\title{
SEPARATING TWO COMPACT SETS BY A PARALLELOTOPE
}

\author{
STEVEN R. LAY
}

\begin{abstract}
Let $\boldsymbol{P}$ and $Q$ be compact subsets of Euclidean $n$-space. Paul Kirchberger has established that there exists a closed half-space $M$ such that $P \subset M$ and $Q \cap M=\varnothing$ iff for each set $S$ consisting of $n+2$ or fewer points of $P \cup Q$, there exists a closed half-space $M_{S}$ such that $(P \cap S) \subset M_{S}$ and $(Q \cap S) \cap M_{S}=\varnothing$. In this paper the problem of replacing the half-spaces by parallelotopes is considered, and the critical number of points in $P \cup Q$ is shown to be $n+1$. Applications of this are drawn to systems of linear inequalities and to Caratheodory's theorem.
\end{abstract}

The question of separating two compact sets by various geometric figures has been of interest since the turn of the century. The first combinatorial result in this direction was established by Paul Kirchberger [2] in 1903 when he proved (in a slightly different form): "Suppose $P$ and $Q$ are nonempty compact subsets of Euclidean space $E^{n}$. Then there exists a closed half-space $M$ such that $P \subset M$ and $Q \cap M=\varnothing$ iff for each set $S$ consisting of $n+2$ or fewer points of $P \cup Q$, there exists a closed half-space $M_{S}$ such that $(P \cap S) \subset M_{S}$ and $(Q \cap S) \cap M_{S}=\varnothing$."

The problem of replacing the half-spaces by other geometric figures was posed by F. A. Valentine [5] in 1964. For results pertaining to spheres and $k$-cylinders, see Lay ([3], [4]). (A $k$-cylinder is the algebraic sum of a compact convex set and a $k$-dimensional subspace.) In this paper we shall investigate the problem of replacing the half-spaces by parallelotopes. This type of separation is particularly useful because the separating set can be described by a system of simultaneous linear inequalities. It also enables us to derive a strengthened form of the classical Carathéodory Theorem.

1. Definitions and notation. Given a basis $\beta \equiv\left\{b_{1}, b_{2}, \ldots, b_{n}\right\}$ for $E^{n}$, let $H_{i}$ $(i=1, \ldots, n)$ be the $(n-1)$-dimensional subspace spanned by $\beta \sim\left\{b_{i}\right\}$. A $\beta$-box is a (possibly degenerate) compact solid parallelotope in which each side is parallel to one of the $H_{i}(i=1, \ldots, n)$.

Algebraically, for any point $x \equiv \alpha_{1} b_{1}+\cdots+\alpha_{n} b_{n}$ we define the $i$ th coordinate function $\pi_{i}$ by $\pi_{i}(x)=\alpha_{i}$. Then the null space of $\pi_{i}$ is $H_{i}$ and a $\beta$-box is the set of all points $x$ that satisfy a system of linear inequalities of the form

Received by the editors June 22, 1979.

AMS (MOS) subject classifications (1970). Primary 52A20, 52A35.

Key words and phrases. Separation of compact sets, parallelotope, Kirchberger's theorem, Caratheodory's theorem. 


$$
\begin{gathered}
m_{1}<\pi_{1}(x)<M_{1} \\
m_{2}<\pi_{2}(x)<M_{2} \text { for real numbers } m_{i}<M_{i}(i=1, \ldots, n) . \\
\quad: \\
m_{n}<\pi_{n}(x)<M_{n}
\end{gathered}
$$

Given any nonempty compact subset $P$ of $E^{n}$ there is a unique minimal $\beta$-box containing $P$. Specifically, take

$$
m_{i}=\inf _{x \in P} \pi_{i}(x) \text { and } M_{i}=\sup _{x \in P} \pi_{i}(x) \quad(i=1, \ldots, n) .
$$

The $\beta$-box so determined clearly contains $P$ and is contained in any other $\beta$-box containing $P$.

2. ThEOREM. Let $P$ and $Q$ be nonempty compact subsets of $E^{n}(n>2)$. Then for any basis $\beta$ of $E^{n}$ the following three conditions are equivalent:

(1) There exists $a \beta$-box $B$ such that $P \subset B$ and $Q \cap B=\varnothing$.

(2) For each subset $S$ of $n+1$ or fewer points of $P \cup Q$, there exists a $\beta$-box $B_{S}$ such that $(P \cap S) \subset B_{S}$ and $(Q \cap S) \cap B_{S}=\varnothing$.

(3) For each subset $T$ of $n$ or fewer points of $P$, the minimal $\beta$-box containing $T$ is disjoint from $Q$.

Proof. The implication (1) $\Rightarrow(2)$ is obvious. To see that (2) implies (3), suppose that (3) does not hold. Then there exists a subset $T$ of $n$ or fewer points of $P$ such that $Q$ intersects the minimal $\beta$-box $B_{T}$ containing $T$. Let $q \in Q \cap B_{T}$. Then $S \equiv\{q\} \cup T$ is a subset of $n+1$ or fewer points of $P \cup Q$ such that there is no $\beta$-box containing $P \cap S=T$ and disjoint from $Q \cap S=\{q\}$. Thus condition (2) does not hold and we have proved the contrapositive of $(2) \Rightarrow(3)$.

The proof that (3) implies (1) is by an induction on the dimension $n$ of the space. Suppose that $n>2$ and that the theorem holds for spaces of dimension $m$, where $2<m<n$. Suppose further that condition (1) does not hold. Then the minimal $\beta$-box $B$ containing $P$ is not disjoint from $Q$. That is, there exists a point $q$ in $Q \cap B$. We must show that condition (3) does not hold.

Define a projection mapping $f$ from $E^{n}$ onto $E^{n-1}$ by

$$
f\left(\alpha_{1} b_{1}+\alpha_{2} b_{2}+\cdots+\alpha_{n} b_{n}\right)=\alpha_{2} b_{2}+\cdots+\alpha_{n} b_{n} .
$$

Then $\beta^{\prime} \equiv\left\{b_{2}, \ldots, b_{n}\right\}$ is a basis for $E^{n-1}$ and $f(B)$ is the minimal $\beta^{\prime}$-box containing $f(P)$. Now $f(q) \in f(B)$, so the theorem applied to the compact sets $f(P)$ and $f(q)$ in $E^{n-1}$ implies there exists a subset $T^{\prime}$ of $n-1$ or fewer points of $f(P)$ such that $f(q)$ is in the minimal $\beta^{\prime}$-box $B_{T^{\prime}}$ containing $T^{\prime}$. Let $T$ be a subset of $n-1$ or fewer points of $P$ such that $f(T)=T^{\prime}$. Since $f(q) \in B_{T^{\prime}}$ and $\pi_{i} \circ f=\pi_{i}$ for $i=2, \ldots, n$, it follows that

$$
\inf _{x \in T} \pi_{i}(x)<\pi_{i}(q)<\sup _{x \in T} \pi_{i}(x)
$$

for $i=2, \ldots, n$. If these inequalities also hold for $i=1$, then $q$ is in the minimal $\beta$-box $B_{T}$ containing $T$, and we are done. If they do not hold for $i=1$, then either 


$$
\pi_{1}(q)<\inf _{x \in T} \pi_{1}(x) \text { or } \pi_{1}(q)>\sup _{x \in T} \pi_{1}(x) .
$$

If $\pi_{1}(q)<\inf _{x \in T} \pi_{1}(x)$, then since $P$ is compact we may choose a point $p$ in $P$ such that $\pi_{1}(p)=\inf _{x \in P} \pi_{1}(x)$. Since $q \in B$ we have $\pi_{1}(q)>\inf _{x \in P} \pi_{1}(x)$ and it follows that

$$
\inf _{x \in T \cup\{p\}} \pi_{1}(x)<\pi_{1}(q)<\sup _{x \in T \cup\{p\}} \pi_{1}(x) .
$$

Similarly, if $\pi_{1}(q)>\sup _{x \in T} \pi_{1}(x)$ we choose a point $p$ in $P$ such that $\pi_{1}(p)=$ $\sup _{x \in P} \pi_{1}(x)$ and obtain the same result. Thus in both cases we have found a subset of $n$ or fewer points of $P$ whose minimal $\beta$-box contains the point $q$. Therefore condition (3) does not hold and we have established the induction part of the proof.

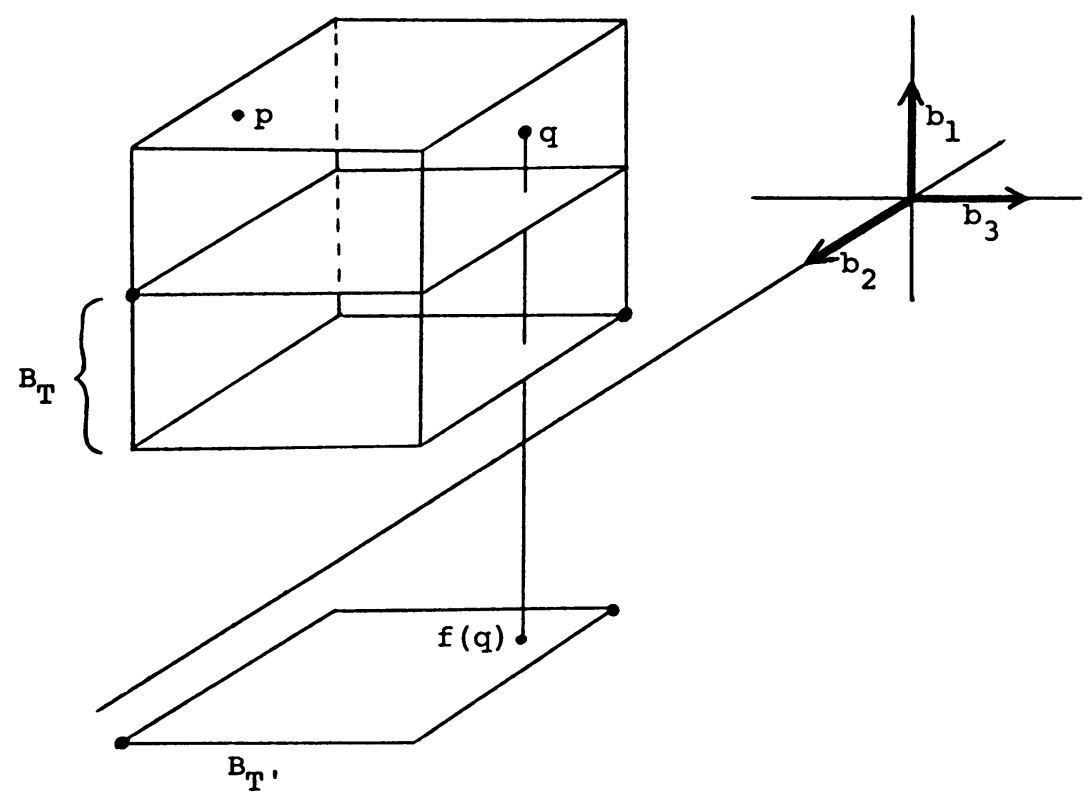

FIGURE 1

Finally we must show that (3) implies (1) when $n=2$. Assume that condition (3) holds and let $B$ be the minimal $\beta$-box containing $P$. Since $B$ is minimal we may select a point of $P$ on each of the four sides of the parallelogram $B$. Call these points $p_{1}, p_{2}, p_{3}$, and $p_{4}$. (They are not necessarily unique or distinct.) Suppose that there exists a point $q$ in $Q \cap B$. We must find two points of $P$ such that $q$ lies in the minimal $\beta$-box containing those two points. Now for each pair of points $p_{i}$ and $p_{j}(i, j=1,2,3,4)$, let $B_{i j}$ be the minimal $\beta$-box containing $\left\{p_{i}, p_{j}\right\}$. Then it is easy to see that $B$ is the union of all the $B_{i j}$ 's. In fact, $B$ can be expressed as the union of five or fewer of the $B_{i j}$ 's. For example, see Figure 2 below. Thus $q \in B_{i j}$ for some particular $i$ and $j$. This contradicts our assumption that condition (3) holds and so $Q$ and $B$ must be disjoint. 


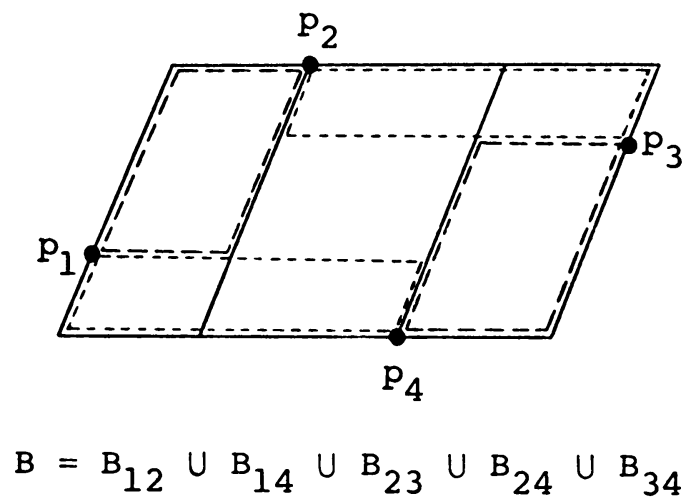

FIGURE 2

3. Applications. If we rephrase the theorem in terms of systems of inequalities we obtain the following corollary.

COROLlaRY 1. Let $P$ be a nonempty compact subset of $E^{n}(n \geqslant 2)$ and suppose that a point $q$ in $E^{n}$ satisfies the following system of inequalities:

$$
\inf _{x \in P} \pi_{i}(x) \leqslant \pi_{i}(q) \leqslant \sup _{x \in P} \pi_{i}(x) \text { for } i=1, \ldots, n .
$$

Then there exists a subset $T$ of $n$ or fewer points of $P$ such that

$$
\inf _{x \in T} \pi_{i}(x) \leqslant \pi_{i}(q) \leqslant \sup _{x \in T} \pi_{i}(x) \text { for } i=1, \ldots, n .
$$

It is not surprising in Corollary 1 that there exists a finite subset $T$ with the desired property. Indeed, we can choose a maximizing point and a minimizing point in $P$ for each coordinate and readily obtain a subset $T$ of $2 n$ points in $P$ which work. The force of the corollary is that there exist $n$ points in $P$ with the same property.

There is a special case included in the theorem which is of particular interest because of its similarity to the classical theorem of Carathéodory [1]:

"Let $P$ be a compact subset of $E^{n}$ and let $q$ be a point in $E^{n}$. Suppose that given any subset $T$ of $n+1$ or fewer points of $P$ there exists a hyperplane which strictly separates $T$ and $q$. Then $q$ is not in the convex hull of $P$."

In the statement of this theorem we understand that a hyperplane $H$ is said to strictly separate $T$ and $q$ if $T$ lies in one of the open half-spaces determined by $H$ and $q$ lies in the other. The following corollary shows that if the orientation of the separating hyperplanes is properly restricted, then it suffices to consider subsets of $n$ or fewer points of $P$.

COROllaRY 2. Let $P$ be a nonempty compact subset of $E^{n}(n \geqslant 2)$ and let $q$ be a point in $E^{n}$. Given a basis $\beta \equiv\left\{b_{1}, \ldots, b_{n}\right\}$ of $E^{n}$, let $H_{i}(i=1, \ldots, n)$ be the $(n-1)$-dimensional subspace spanned by $\beta \sim\left\{b_{i}\right\}$. Suppose that given any subset $T$ of $n$ or fewer points of $P$ there exists a hyperplane which is parallel to one of the $H_{i}$ $(i=1, \ldots, n)$ and which strictly separates $T$ and $q$. Then $q$ is not in the convex hull of $P$. 
Proof. The hypotheses imply that for each subset $T$ of $n$ or fewer points of $P$ there exists a $\beta$-box containing $T$ and disjoint from $\{q\}$. Applying the theorem we conclude that there exists a $\beta$-box containing $P$ and disjoint from $\{q\}$, and so $q$ is not in the convex hull of $P$.

4. Remarks. In the proof of the main theorem, it is crucial that all of the parallelotopes are similarly oriented. What happens if this restriction is dropped? It turns out that under these conditons there is no Kirchberger-type number. Specifically, given any positive integer $k$, there exist nonempty compact subsets $P$ and $Q$ of $E^{2}$ with the following property:

Given any subset $T$ of $k$ or fewer points of $P$, there exists a parallelogram containing $T$ which is disjoint from $Q$. But there is no parallelogram containing all of $P$ which is disjoint from $Q$.

We may construct $P$ and $Q$ in the following way.

Let $m$ be an even number $\geqslant k$ and let $P$ be the vertices of a regular $(m+1)$-gon centered at the origin. (See Figure 3 for $m=6$.) Suppose one point, say $x$, of $P$ is omitted. Let $R_{1}$ be a circumscribing rectangle around the remaining points such that one side of $R_{1}$ contains the edge of the original polygon which is opposite to $x$. Let $r_{1}$ be the distance from the origin to the farthest corner (point $b$ ) of $R_{1}$.

Now let $R_{2}$ be a rectangle circumscribing all of $P$ which is oriented similarly to $R_{1}$. Let $r_{2}$ be the distance from the origin to the farthest corner (point $c$ ) of $R_{2}$. Then it is clear that $r_{2}>r_{1}$. (The two triangles $\overline{o x c}$ and $\overline{o a b}$ have equal bases $\overline{a b}$ and $\overline{x c}$. Since the altitude of the former triangle is longer than the latter's, so is its hypotenuse.) Furthermore, from symmetry considerations it follows that any parallelogram circumscribing $P$ will have a corner at least as far as $r_{2}$ from the origin. If $r$ is a positive number satisfying

$$
r_{1}<r<r_{2}
$$

then the circle $Q$ of radius $r$ centered at the origin will then have the required properties.
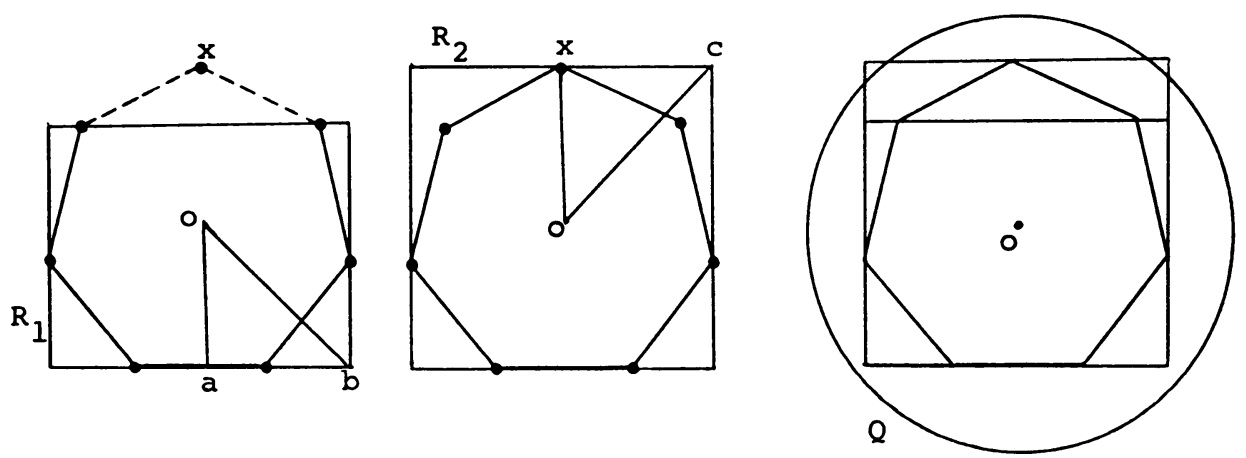

Figure 3 


\section{BIBLIOGRAPHY}

1. C. Carathéodory, Über den Variabilitätsbereich der Koeffizienten von Potenzreihen, die gegebene Werte nicht annahmen, Math. Ann. 4 (1907), 95-115.

2. P. Kirchberger, Uber Tschebyschefsche Annaherungsmethoden, Math. Ann. 57 (1903), 509-540.

3. S. R. Lay, On separation by spherical surfaces, Amer. Math. Monthly 78 (1971), 1112-1113.

4. __ Separation by cylindrical surfaces, Proc. Amer. Math. Soc. 36 (1972), 224-228.

5. F. A. Valentine, Convex sets, McGraw-Hill, New York, 1964, p. 177.

Department of Mathematics, Aurora College, Aurora, illinois 60507 